\title{
BRUHAT ORDER AND NIL-HECKE ALGEBRAS FOR WEYL GROUPOIDS
}

\author{
IVÁN ANGIONO AND HIROYUKI YAMANE
}

\begin{abstract}
We introduce nil-Hecke algebras for Weyl groupoids. We describe a basis and some properties of these algebras which lead to a notion of Bruhat order for Weyl groupoids.
\end{abstract}

\section{INTRODUCTION}

One of the remarkable points of contragredient Lie superalgebras, which makes a difference with Lie algebras, is the existence of different matrices and parities on the generators whose corresponding Lie superalgebras are isomorphic. These algebras are related by odd reflections $[\mathrm{Se}$ but then a problem arises: how to manage these reflections together with the Weyl group (of the even part) at the same time. By [SV] the Grothendieck ring of these Lie superalgebras is obtained as invariant of a super Weyl groupoid. The Weyl groupoid considered here seems to be related with their super Weyl groupoids.

A similar context appears for Nichols algebras of diagonal type, which are generalizations of (the positive part of) quantized enveloping algebras and Frobenius-Lusztig kernels. The existence of Weyl groupoid for Nichols algebras was started in [H1] for diagonal braidings and continued in [AHS] in a more general context. An axiomatic study was initiated in [HY1, where a Matsumoto-Tits type theorem was given and a relation with Lie superalgebras was proposed. Indeed every contragredient Lie superalgebra has a Weyl groupoid by [AA], see also [AY for characteristic 0 case. Some braidings of diagonal type are related with contragredient Lie superalgebras over fields of characteristic zero [AAY, but this relation can be extended to positive characteristic [AA since a Weyl groupoid can be attached to any (finite-dimensional) contragredient Lie superalgebra.

This Weyl groupoid presents several applications. In HY2 Heckenberger and the second author obtained the factorization formula of the Shapovalov determinants of the generalized quantum groups $U$, where an example of $U$ is any Frobenius-Lusztig kernel (i.e., Lusztig's small quantum group); they used an action of the Weyl groupoid on Verma modules of $U$. Any reduced expression of its longest element gives an explicit realization of the universal R-matrix of $U$ AY]. A special reduced expression of it was used to classify finite dimensional irreducible representations of $U$ AYY. A weak order of the Weyl groupoids

2010 Mathematics Subject Classification. 16T05, Secondary 17B37.

The work of I. A. was partially supported by CONICET, FONCyT-ANPCyT, Secyt (UNC). The work of H. Y. was partially supported by Japan's Grand-in-Aid for Scientific Research (C) 25400040 . 
turned out to be useful since it is used in the classification of the coideals of Nichols algebras [HS. These orders are related with convex orders on the positive roots $[\mathrm{A}$, a notion generalizing the one for classical root systems. In [HW] topological structures were studied, involving hyperplanes and associated with Weyl groupoids. It was used the left order of the Weyl groupoid.

Then one can naturally ask if there exists an analogous of Bruhat order for Weyl groupoids.

The Bruhat order of Coxeter groups essentially appears in various areas in mathematics, for example in so-called Schubert calculus treated for studying the cohomology of a flag manifold [BS]. Another example is the formula given in [IN, Proposition 2.2], a key result to concretely calculate the value of the equivariant Schubert class.

In this paper, we introduce a Bruhat order, or a strong order, for the elements of the Weyl groupoids. To this end we also introduce nil-Hecke algebras for Weyl groupoids and obtain some properties. A future work is to establish the existence of Kazhdan-Lusztig polynomials of Weyl groupoids and the applications of these results to the representation theory of Nichols algebras.

This paper is organized as follows. We give a brief introduction of Bruhat orders for Coxeter groups in Section 2 including the main properties. Then in Section 3 we recall the definition of basic datum, Coxeter groupoid and generalized root systems. In Section 4 we introduce the nil-Hecke algebras for Weyl groupoids. We describe a basis indexed by the elements of the Weyl groupoid and a representation of a groupoid covering the Weyl groupoid on the nil-Hecke algebra. Finally we introduce the definition of Bruhat order in Section 5 and prove the independence of the reduced expression.

Notation. For $\theta \in \mathbb{N}$, let $\mathbb{I}_{\theta}=\{1,2, \ldots, \theta\}$, or simply $\mathbb{I}$ if $\theta$ is clear from the context. Let $\left\{\alpha_{i}\right\}_{i \in \mathbb{I}}$ be the canonical basis of $\mathbb{Z}^{I}$. Also, $\mathbb{K}$ will denote a commutative ring.

\section{Bruhat order for COXETER GRoups}

We start by recalling the definition the Bruhat order of Coxeter groups and we outline a proof about his good definition using Nil-Hecke algebras.

A Coxeter matrix is a symmetric matrix $M=\left(m_{i j}\right)_{i, j \in \mathbb{I}}$ with entries in $\mathbb{N} \cup\{+\infty\}$ such that $m_{i i}=1$ and $m_{i j} \geq 2$, for all $i \neq j \in \mathbb{I}$. The Coxeter system of $M$ is the pair $(W, S)$, where $W$ is the group presented by generators $S=\left\{s_{1}, \ldots, s_{\theta}\right\}$ and relations $\left(s_{i} s_{j}\right)^{m_{i j}}=e$, for all $i, j \in \mathbb{I}$.

Definition 2.1. Let $(W, S)$ be a Coxeter system. Given $w, w^{\prime} \in W$, we say that $w^{\prime}<w$ if there exists a reduced expression $w=s_{1} s_{2} \cdots s_{n}$ and a subsequence $i_{1}, i_{2}, \ldots, i_{r}$ of $1,2, \ldots, n$ such that $w^{\prime}=s_{i_{1}} s_{i_{2}} \cdots s_{i_{r}}$ is a reduced expression of $w^{\prime}$. It defines a partial order on $W$ called the Bruhat order.

Theorem 2.2. Let $w, w^{\prime} \in W$ be such that $w^{\prime}<w$. Then for any reduced expression $w=t_{1} t_{2} \cdots t_{n}, t_{k} \in S$, there exists a subsequence $i_{1}, i_{2}, \ldots, i_{r}$ of $1,2, \ldots, r$ such that $w^{\prime}=t_{i_{1}} t_{i_{2}} \cdots t_{i_{r}}$ is a reduced expression of $w^{\prime}$.

There exists a proof involving the nil-Hecke algebra of $(W, S)$ when $W$ is a Weyl group. Let us explain it. Let $\mathcal{N}$ be a free left $\mathbb{K}$-module with basis $n_{w}$, 
$w \in W$. Hence $\mathcal{N}$ is a $\mathbb{K}$-algebra with multiplication

$$
n_{w} n_{w^{\prime}}:= \begin{cases}n_{w w^{\prime}} & \text { if } \ell\left(w w^{\prime}\right)=\ell(w)+\ell\left(w^{\prime}\right) \\ 0 & \text { otherwise }\end{cases}
$$

The algebra $\mathcal{N}$ is called the Nil-Hecke algebra.

Let $\Delta$ be a root system associated to $(W, S)$. Let $\Pi=\left\{\alpha_{i} \mid i \in I\right\}$ be the basis of $\Delta, \Delta^{+}:=\Delta \cup \mathbb{N}_{0} \Pi$. Then $\Delta=\Delta^{+} \cup-\Delta^{+}$.

Assume $\mathbb{K}$ is the free additive group with basis $\Pi$. Let $n_{i}:=n_{s_{i}} \in \mathcal{N}$. For $\lambda \in \mathbb{K}$, let $h_{i}(\lambda):=1+\lambda n_{i}$. Given $s_{i_{1}} \cdots s_{i_{m}}$ a reduced expression of $w \in W$, define $h(w) \in \mathcal{N}$ by

$$
h(w):=h_{i_{1}}\left(\alpha_{i_{1}}\right) h_{i_{2}}\left(s_{i_{1}}\left(\alpha_{i_{2}}\right)\right) \cdots h_{i_{\ell(w)}}\left(s_{i_{1}} \cdots s_{i_{\ell(w)-1}}\left(\alpha_{i_{\ell(w)}}\right)\right) .
$$

It follows that $h(w)$ is independent of the choice of a reduced expression of $w$. This is proved by direct computation for rank-two Coxeter groups, and the general proof holds since the defining relations involve only two letters of $S$. Hence the theorem follows since

$$
\alpha_{i_{1}}, s_{i_{1}}\left(\alpha_{i_{2}}\right), \ldots, s_{i_{1}} \cdots s_{i_{\ell(w)-1}}\left(\alpha_{i_{\ell(w)}}\right) \in \mathbb{N}_{0} \Pi .
$$

\section{WEYL GROUPOIDS AND GENERALIZED ROOT SYSTEMS}

We follow the notation of $\mathrm{AA}$, see also $[\mathrm{CH}, \mathrm{HY} 1$.

3.1. Basic data and Coxeter groupoids. Given $\theta \in \mathbb{N}, \mathbb{I}=\mathbb{I}_{\theta}, \mathcal{X} \neq \emptyset$ a non-empty set and $\rho: \mathbb{I} \rightarrow \mathbb{S}_{\mathcal{X}}$, the pair $(\mathcal{X}, \rho)$ is a basic datum of base $|\mathcal{X}|$ and size $\mathbb{I}$ if $\rho_{i}^{2}=$ id for all $i \in \mathbb{I}$.

We denote by $\mathcal{Q}_{\rho}$ the quiver with vertices $\mathcal{X}$ and arrows

$\sigma_{i}^{x}:=\left(x, i, \rho_{i}(x)\right), i \in \mathbb{I}, x \in \mathcal{X}, \quad$ with target $t\left(\sigma_{i}^{x}\right)=x$, source $s\left(\sigma_{i}^{x}\right)=\rho_{i}(x)$.

The object change diagram $[\mathrm{CH}]$, or simply the diagram, of $(\mathcal{X}, \rho)$ is a simplified graphical description of $\mathcal{Q}_{\rho}$. It is the graph with bullets labeled with $\mathcal{X}$ and one arrow between $x \neq y$, decorated with the label $i$, for each pair $(x, i, y)$, $(y, i, x)$ such that $\rho_{i}(x)=y$. Therefore the loops at a vertex $x$ are deduced from the diagram and $\theta .(\mathcal{X}, \rho)$ is connected if $\mathcal{Q}_{\rho}$ is connected.

For any quotient of the free groupoid $F\left(\mathcal{Q}_{\rho}\right), \sigma_{i_{1}}^{x} \sigma_{i_{2}} \cdots \sigma_{i_{t}}$ means

$$
\sigma_{i_{1}}^{x} \sigma_{i_{2}}^{\rho_{i_{1}}(x)} \cdots \sigma_{i_{t}}^{\rho_{i_{t-1}} \cdots \rho_{i_{1}}(x)}
$$

omitting the implicit superscripts, uniquely determined to have compositions.

Given a basic datum $(\mathcal{X}, \rho)$, a Coxeter datum is a triple $(\mathcal{X}, \rho, \mathbf{M})$, where $\mathbf{M}=\left(\mathbf{m}^{x}\right)_{x \in \mathcal{X}}, \mathbf{m}^{x}=\left(m_{i j}^{x}\right)_{i, j \in \mathbb{I}}$, is a family of Coxeter matrices such that

$$
s\left(\left(\sigma_{i}^{x} \sigma_{j}\right)^{m_{i j}^{x}}\right)=x, \quad \text { for all } i, j \in \mathbb{I} \text { and } x \in \mathcal{X} .
$$

The Coxeter groupoid $\mathcal{W}(\mathcal{X}, \rho, \mathbf{M})$ [HY1, Definition 1] is the groupoid generated by the quiver $\mathcal{Q}_{\rho}$ with relations

$$
\left(\sigma_{i}^{x} \sigma_{j}\right)^{m_{i j}^{x}}=\mathrm{id}_{x}, \quad i, j \in \mathbb{I}, x \in \mathcal{X} .
$$

Notice that either $\sigma_{i}^{x}$ is an involution if $\rho_{i}(x)=x$, or else $\sigma_{i}^{x}$ is the inverse arrow of $\sigma_{i}^{\rho_{i}(x)}$ if $\rho_{i}(x) \neq x$. 
As in [HY1] we denote by $\widetilde{\mathcal{W}}$ the corresponding quotient of the quiver groupoid $\mathcal{Q}_{\rho}$ subject to the same relations as for $\mathcal{W}$ except (2) for $i=j$; that is, we omit the relations $\sigma_{i}^{x} \sigma_{i}^{r_{i}(x)}=\mathrm{e}^{x}$ for $i \in \mathbb{I}, x \in \mathcal{X}$. Then there exists a canonical projection $\mathbb{K} \widetilde{\mathcal{W}} \rightarrow \mathbb{K} \mathcal{W}$ such that $\widetilde{\sigma}_{i}^{x} \mapsto \sigma_{i}^{x}, \widetilde{\mathrm{e}}^{x} \mapsto \mathrm{e}^{x}$.

Remark 3.1. Let $\mathbb{K}$ be a commutative ring with 1 . The groupoid algebra of $\mathcal{W}(\mathcal{X}, \rho, \mathbf{M})$ is generated by $\mathrm{e}^{x}, \sigma_{i}^{x}, x \in \mathcal{X}, i \in \mathbb{I}$, with relations (2) and

$$
\mathrm{e}^{x} \mathrm{e}^{y}=\delta_{x, y} \mathrm{e}^{x}, \quad \sum_{x \in \mathcal{X}} \mathrm{e}^{x}=1, \quad \sigma_{i}^{x} \mathrm{e}^{\rho_{i}(x)}=\mathrm{e}^{x} \sigma_{i}^{x}=\sigma_{i}^{x} .
$$

A covering of a basic datum $(\mathcal{X}, \rho)$ is a 3 -uple $(\widetilde{\mathcal{X}}, \widetilde{\rho}, F)$ such that $(\widetilde{\mathcal{X}}, \widetilde{\rho})$ is a basic datum and $F: \widetilde{\mathcal{X}} \rightarrow \mathcal{X}$ is a surjective map such that $\rho_{i} \circ F=F \circ \widetilde{\rho}_{i}$ for all $i \in \mathbb{I}$.

Given two Coxeter data $(\mathcal{X}, \rho, \mathbf{M}),(\widetilde{\mathcal{X}}, \widetilde{\rho}, \widetilde{\mathbf{M}})$ and $F: \mathcal{X}^{\prime} \rightarrow \mathcal{X}$ such that $(\tilde{\mathcal{X}}, \widetilde{\rho}, F)$ is a covering of $(\mathcal{X}, \rho)$, we say that $F$ is a covering of Coxeter data if $M^{F\left(t_{1}\right)}=M^{x}$ for all $x \in \widetilde{\mathcal{X}}$. In such a case $F$ induces a canonical surjective map of groupoids $F: \mathcal{W}(\widetilde{\mathcal{X}}, \widetilde{\rho}, \widetilde{\mathbf{M}}) \rightarrow \mathcal{W}(\mathcal{X}, \rho, \mathbf{M})$. It is a particular case of a morphism between Coxeter data $\mathrm{AA}$.

3.2. Generalized root systems and Weyl groupoids. Recall that $C=$ $\left(c_{i j}\right) \in \mathbb{Z}^{\theta \times \theta}$ is a generalized Cartan matrix $\mathrm{Ka}$ if for all $i \neq j \in \mathbb{I}, c_{i i}=2$, $c_{i j} \leq 0$, and $c_{i j}=0$ if and only if $c_{j i}=0$.

A semi-Cartan graph is a triple $(\mathcal{X}, \rho, \mathcal{C})$ such that $(\mathcal{X}, \rho)$ is a basic datum of size $\mathbb{I}$ and $\mathcal{C}=\left(C^{x}\right)_{x \in \mathcal{X}}, C^{x}=\left(c_{i j}^{x}\right)_{i, j \in \mathbb{I}}, x \in \mathcal{X}$, is a bundle of generalized Cartan matrices satisfying

$$
c_{i j}^{x}=c_{i j}^{\rho_{i}(x)} \quad \text { for all } x \in \mathcal{X}, i, j \in \mathbb{I} .
$$

We define $s_{i}^{x} \in G L_{\theta}(\mathbb{Z})$ by

$$
s_{i}^{x}\left(\alpha_{j}\right)=\alpha_{j}-c_{i j}^{x} \alpha_{i}, \quad j \in \mathbb{I}, \quad i \in \mathbb{I}, x \in \mathcal{X} .
$$

Then $s_{i}^{x}$ is the inverse of $s_{i}^{\rho_{i}(x)}$ by (3).

Definition 3.2. [HY1, Definition 1] Let $(\mathcal{X}, \rho, \mathcal{C})$ be a semi-Cartan graph. A generalized root system (for short a GRS) is a collection $\mathcal{R}:=\mathcal{R}(\mathcal{X}, \rho, \mathcal{C}, \Delta)$, where $\Delta=\left(\Delta^{x}\right)_{x \in \mathcal{X}}$ is a family of subsets $\Delta^{x} \subset \mathbb{Z}^{\mathbb{I}}$ such that for all $x \in \mathcal{X}$ and all $i \neq j \in \mathbb{I}$,

- $\Delta^{x}=\Delta_{+}^{x} \cup \Delta_{-}^{x}$, where $\Delta_{ \pm}^{x}:= \pm\left(\Delta^{x} \cap \mathbb{N}_{0}^{I}\right) \subset \pm \mathbb{N}_{0}^{I}$,

- $\Delta^{x} \cap \mathbb{Z} \alpha_{i}=\left\{ \pm \alpha_{i}\right\}$

- $s_{i}^{x}\left(\Delta^{x}\right)=\Delta^{\rho_{i}(x)}$,

- $\left(\rho_{i} \rho_{j}\right)^{m_{i j}^{x}}(x)=x$, where $m_{i j}^{x}:=\left|\Delta^{x} \cap\left(\mathbb{N}_{0} \alpha_{i}+\mathbb{N}_{0} \alpha_{j}\right)\right|$.

$\Delta_{+}^{x}$, respectively $\Delta_{-}^{x}$, are called the set of positive, respectively negative, roots. The Weyl groupoid of $\mathcal{R}$ is $\mathcal{W}=\mathcal{W}(\mathcal{X}, \rho, \mathcal{C})$. It is called finite if $R^{x}$ is finite for some $x \in \mathcal{X}$, or equivalently, for all $x \in \mathcal{X}$; see [CH] for other equivalences.

Remark 3.3. Let $(\mathcal{X}, \rho, \mathcal{C})$ be a semi-Cartan graph with generalized root system $\mathcal{R}$. Then the Weyl groupoid is a Coxeter groupoid [HY1]; more precisely, $\mathcal{W}(\mathcal{X}, \rho, \mathcal{C})=\mathcal{W}(\mathcal{X}, \rho, \mathbf{M})$, where $m_{i j}^{x}$ are defined as above for all $i, j \in \mathbb{I}, x \in \mathcal{X}$. 
For any $x \in \mathcal{X}$, the sets of real roots at $x$ is

$$
\left(\Delta^{\mathrm{re}}\right)^{x}=\bigcup_{y \in \mathcal{X}}\left\{w\left(\alpha_{i}\right): i \in \mathbb{I}, w \in \mathcal{W}(y, x)\right\} .
$$

Notice that $w\left(\Delta^{x}\right)=\Delta^{y}$ for all $w \in \mathcal{W}(x, y)$. A semi-Cartan graph $(\mathcal{X}, \rho, \mathcal{C})$ is Cartan if $\Delta^{x}=\bigcup_{y \in \mathcal{X}}\left\{w\left(\alpha_{i}\right): i \in \mathbb{I}, w \in \mathcal{W}(y, x)\right\}$ is a finite GRS.

The notion of covering of Coxeter data $(\mathcal{X}, \rho, \mathbf{M}),(\widetilde{\mathcal{X}}, \widetilde{\rho}, \widetilde{\mathbf{M}})$ extend canonically to semi-Cartan graphs and GRS by requiring, respectively, that $C^{F(x)}=$ $C^{x}$ and $\Delta^{F(x)}=\Delta^{x}$ for all $x \in \widetilde{\mathcal{X}}$.

Example 3.4. A semi-Cartan (or Cartan) graph $(\mathcal{X}, \rho, \mathcal{C})$ is called standard if all the matrices $C^{x}, x \in \mathcal{X}$, are equal. Let $(\mathcal{X}, \rho, \mathcal{C})$ be a standard Cartan graph with root system $\mathcal{R}$. Then $\mathcal{R}$ is finite if and only if each $C^{x}$ is of finite type, in which case each $\Delta^{x}$ is the root system of $C^{x}$.

Finite standard root systems with one point are Weyl groups, of type $A_{2}, B_{2}$ or $G_{2}$. Some other finite standard Weyl groupoids are close to Lie superalgebras, and then with Nichols algebras of super type, see [AAY]. For example, the following Cartan graphs for $\theta=2$ :

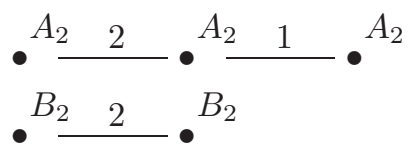

4. Nil Hecke Algebras for Weyl groupoids of Finite Rank

4.1. Basic properties of nil Hecke algebras. We assume that $\mathcal{X}$ is finite. We define a nil-Hecke algebra for each Weyl groupoid, which generalizes the corresponding notion for Weyl groups. Next we prove the first properties of this algebra. In particular we describe a basis using methods close to those for nil-Hecke algebras over Weyl groups.

Definition 4.1. Let $\mathbb{K}$ be a commutative ring with 1 . Let $(\mathcal{X}, \rho, \mathcal{C})$ be a semiCartan graph and $\mathcal{R}$ a GRS of $(\mathcal{X}, \rho, \mathcal{C})$. The nil-Hecke algebra of $(\mathcal{X}, \rho, \mathcal{C})$ is the $\mathbb{K}$-algebra $\mathcal{N}=\mathcal{N}(\mathcal{X}, \rho, \mathcal{C})$ generated by $\mathrm{e}^{x}, n_{i}^{x}, x \in \mathcal{X}, i \in I$, with relations

$$
\begin{aligned}
\mathrm{e}^{x} \mathrm{e}^{y} & =\delta_{x, y} \mathrm{e}^{x}, & \sum_{x \in \mathcal{X}} \mathrm{e}^{x}=1, \\
\underbrace{n_{i}^{x} n_{j} n_{i} \ldots}_{m_{i j}^{x}} & =\underbrace{n_{i}(x)}_{m_{i j}^{x}}=\mathrm{e}^{x} n_{i}^{x}=n_{i}^{x}, & n_{i}^{\rho_{i}(x)} n_{i}^{x}=0,
\end{aligned}
$$

for all $x, y \in \mathcal{X}, i \neq j \in I$.

Remark 4.2. There exists a surjective map $\Upsilon: \mathbb{K} \widetilde{\mathcal{W}} \rightarrow \mathcal{N}$ given by $\widetilde{\mathrm{e}}^{x} \mapsto \mathrm{e}^{x}$, $\tilde{\sigma}_{i}^{x} \mapsto n_{i}^{x}$, for all $x \in \mathcal{X}, i \in I$.

We use a strategy close to the one in [M, Section 4.1] to produce a basis of this algebra. Let $E^{x}, L_{i}^{x}: \mathbb{K} \mathcal{W} \rightarrow \mathbb{K} \mathcal{W}, x \in \mathcal{X}, i \in I$, be the linear maps

$$
E^{x}(w):= \begin{cases}w, & w \in \operatorname{Hom}(\mathcal{W}, x) \\ 0, & \text { otherwise }\end{cases}
$$




$$
L_{i}^{x}(w):= \begin{cases}\sigma_{i}^{x} w, & \sigma_{i}^{x} w \neq 0, \ell\left(\sigma_{i}^{x} w\right)>\ell(w) \\ 0, & \text { otherwise. }\end{cases}
$$

Lemma 4.3. There exists an algebra map $\Lambda: \mathcal{N} \rightarrow \operatorname{End}(\mathbb{K} \mathcal{W})$ such that

$$
\mathrm{e}^{x} \mapsto E^{x}, \quad n_{i}^{x} \mapsto L_{i}^{x}, \quad x \in \mathcal{X}, i \in I .
$$

Proof. It follows that

$$
\sum_{x \in \mathcal{X}} E^{x}=\operatorname{id}_{V}, \quad E^{x} E^{y}=\delta_{x, y} E^{x}, \quad L_{i}^{x} E^{\rho_{i}(x)}=E^{x} L_{i}^{x}=L_{i}^{x},
$$

for all $x, y \in \mathcal{X}, i \in \mathbb{I}$. Also, if $w \in \operatorname{Hom}(\mathcal{W}, x)$ is such that $\ell\left(\sigma_{i}^{\rho_{i}(x)} w\right)>\ell(w)$, then $\sigma_{i}^{\rho_{i}(x)} w \in \operatorname{Hom}\left(\mathcal{W}, \rho_{i}(x)\right)$ satisfies $\sigma_{i}^{x} \sigma_{i}^{\rho_{i}(x)} w=w$, so $L_{i}^{x} L_{i}^{\rho_{i}(x)}(w)=0$; if $\ell\left(\sigma_{i}^{\rho_{i}(x)} w\right)>\ell(w)$, then $L_{i}^{\rho_{i}(x)}(w)=0$ by definition. Hence $L_{i}^{x} L_{i}^{\rho_{i}(x)}=0$. As

$$
\underbrace{\sigma_{i}^{x} \sigma_{j} \sigma_{i} \ldots}_{m_{i j}^{x}}=\underbrace{\sigma_{j}^{x} \sigma_{i} \sigma_{j} \ldots}_{m_{i j}^{x}},
$$

we deduce that

$$
\underbrace{L_{i}^{x} L_{j} L_{i} \ldots}_{m_{i j}^{x}}=\underbrace{L_{j}^{x} L_{i} L_{j} \cdots}_{m_{i j}^{x}}
$$

Therefore $\Lambda$ is well-defined.

Theorem 4.4. Let $w \in \operatorname{Hom}(y, x), x, y \in \mathcal{X}$ be an element of length $m$. If $w=\sigma_{i_{1}}^{x} \cdots \sigma_{i_{m}}=\sigma_{j_{1}}^{x} \cdots \sigma_{j_{m}}$ are two reduced expressions, then

$$
n_{i_{1}}^{x} n_{i_{2}} \cdots n_{i_{m}}=n_{j_{1}}^{x} n_{j_{2}} \cdots n_{j_{m}} .
$$

Call then $T_{w}$ to this element, and set $T_{\mathrm{id}_{x}}:=\mathrm{e}^{x}$ for all $x \in \mathcal{X}$. Then

$$
\left\{T_{w} \mid w \in \operatorname{Hom}(y, x), x, y \in \mathcal{X}\right\}
$$

is a basis of $\mathcal{N}$.

Proof. We claim that $\Phi: \mathcal{N} \rightarrow \mathbb{K} \mathcal{W}, \Phi(n)=\Lambda(n)(1)$ is an isomorphism. Given an element $w \in \mathcal{W}$ of length $m$, take a reduced expression $w=\sigma_{i_{1}}^{x} \cdots \sigma_{i_{m}}$. Then

$$
\Phi\left(n_{i_{1}}^{x} n_{i_{2}} \cdots n_{i_{m}}\right)=L_{i_{1}}^{x} L_{i_{2}} \cdots L_{i_{m}}(1)=\sigma_{i_{1}}^{x} \sigma_{i_{2}} \cdots \sigma_{i_{m}}=w .
$$

Therefore $\Phi$ is surjective.

To prove the injectivity we fix a reduced expression $w=\sigma_{i_{1}}^{x} \cdots \sigma_{i_{m}}$ for each $w \in \mathcal{W}$. We set $T_{w}^{\prime}:=n_{i_{1}}^{x} n_{i_{2}} \cdots n_{i_{m}}$ if $\ell(w) \geq 1$, and $T_{\mathrm{id}_{x}}:=\mathrm{e}^{x}$ for each $x \in \mathcal{X}$. Let $\mathcal{N}^{\prime}$ be the subspace spanned by $T_{w}^{\prime}, w \in \mathcal{W}$. Note that $1 \in \mathcal{N}^{\prime}$. For each $x, y, z \in \mathcal{X}$ and $w \in \operatorname{Hom}(y, z)$, it holds that $\mathrm{e}^{x} T_{w}^{\prime}=\delta_{x, z} T_{w}^{\prime}$. Given $i \in I$, we compute the product $n_{i}^{x} T_{w}^{\prime}$. If $\ell(w)=0$, that is $w=\mathrm{id}_{y}$ for some $y \in \mathcal{X}$, then $n_{i}^{x} T_{w}^{\prime}=\delta_{x, y} n_{i}^{x} \in \mathcal{N}^{\prime}$. Otherwise, let $w=\sigma_{i_{1}}^{y} \cdots \sigma_{i_{m}}$ be the fixed reduced expression; we consider three possibilities:

- $y \neq \rho_{i}(x): n_{i}^{x} T_{w}^{\prime}=0$. 
- $y=\rho_{i}(x), \ell\left(\sigma_{i}^{x} w\right)=m+1$ : let $v=\sigma_{i}^{x} w$, and $v=\sigma_{j_{0}}^{x} \sigma_{j_{1}} \cdots \sigma_{j_{m}}$ the fixed reduced expression. By [HY1, Theorem 5], we have that

$$
\tilde{\sigma}_{j_{0}}^{x} \tilde{\sigma}_{j_{1}} \cdots \tilde{\sigma}_{j_{m}}=\tilde{\sigma}_{i}^{x} \widetilde{\sigma}_{i_{1}} \cdots \tilde{\sigma}_{i_{m}}
$$

in $\widetilde{\mathcal{W}}$, so applying the morphism $\Upsilon$ in Remark 4.2

$$
n_{i}^{x} T_{w}^{\prime}=n_{i}^{x} n_{i_{1}} n_{i_{2}} \cdots n_{i_{m}}=T_{v}^{\prime} \in \mathcal{N}^{\prime} .
$$

- $y=\rho_{i}(x), \ell\left(\sigma_{i}^{x} w\right)<m+1$ : By [HY1, Corollary 6], there exists $j_{1}, \ldots, j_{m+1} \in \mathbb{I}, t \in\{1, \ldots, m\}$ such that $j_{t}=j_{t+1}$ and

$$
\tilde{\sigma}_{j_{1}}^{x} \widetilde{\sigma}_{j_{2}} \cdots \widetilde{\sigma}_{j_{m+1}}=\widetilde{\sigma}_{i}^{x} \widetilde{\sigma}_{i_{1}} \cdots \widetilde{\sigma}_{i_{m}}
$$

in $\widetilde{\mathcal{W}}$, so by applying the morphism $\Upsilon$ in Remark 4.2,

$$
n_{i}^{x} T_{w}^{\prime}=n_{j_{1}}^{x} n_{j_{1}} \cdots n_{j_{m+1}}=0 .
$$

Then $n_{i}^{x} T_{w}^{\prime} \in \mathcal{N}^{\prime}$, so $\mathcal{N}^{\prime}$ is a left ideal, and we conclude that $\mathcal{N}^{\prime}=\mathcal{N}$; that is, the elements $T_{w}^{\prime}, w \in \mathcal{W}$, $\operatorname{span} \mathcal{N}$. As $\Phi$ apply them to a basis of $\mathbb{K} \mathcal{W}, \Phi$ is an isomorphism.

If we set a different reduced expression $w=\sigma_{j_{1}}^{x} \cdots \sigma_{j_{m}}$, then

$$
\Phi\left(n_{j_{1}}^{x} n_{j_{2}} \cdots n_{j_{m}}\right)=w
$$

by an analogous computation, so (9) follows.

Corollary 4.5. The representation $\Lambda: \mathcal{N} \rightarrow \operatorname{End}(\mathbb{K} \mathcal{W})$ of the nil-Hecke algebra $\mathcal{N}$ of Lemma 4.3 is faithful.

Proof. It follows since the isomorphism $\Phi$ introduced in the previous proof factorizes through $\Lambda$.

The existence of coverings induce algebra maps between the corresponding nil-Hecke algebras.

Corollary 4.6. Let $\left(\mathcal{W}^{\prime}, f\right)$ be a covering of a Weyl groupoid $\mathcal{W}$. Let $\mathcal{N}^{\prime}$ be the nil Hecke algebra of $\mathcal{W}^{\prime}$. There exists an injective algebra map $F: \mathcal{N} \rightarrow \mathcal{N}^{\prime}$ such that

$$
\mathrm{e}^{x} \mapsto \sum_{y \in f^{-1}\left(t_{1}\right)} \mathrm{e}^{y}, \quad \mathrm{n}_{i}^{x} \mapsto \sum_{y \in f^{-1}\left(t_{1}\right)} \mathrm{n}_{i}^{y}, \quad x \in \mathcal{X}, i \in I
$$

Proof. Set $\mathbf{e}^{x}=\sum_{y \in f^{-1}\left(t_{1}\right)} \mathrm{e}^{y}, \mathbf{n}_{i}^{x}=\sum_{y \in f^{-1}\left(t_{1}\right)} \mathrm{n}_{i}^{y}$. Then these elements of $\mathcal{N}^{\prime}$ satisfy the corresponding defining relations (8) of $\mathcal{N}^{\prime}$ for all $x \in \mathcal{X}, i \neq j \in I$, so $F$ is well-defined.

The injectivity is a consequence of Theorem 4.4, because the image of that basis of $\mathcal{N}$ is a linearly independent set in $\mathcal{N}^{\prime}$ by the same Theorem. 
4.2. A useful representation of $\widetilde{\mathcal{W}}$. Set $\mathbb{K}=\mathbb{Z}\left[t_{i} \mid i \in \mathbb{I}\right]$. We identify the additive subgroup $\oplus_{i \in \mathbb{I}} \mathbb{Z} t_{i}$ of $\mathbb{K}$ with $\mathbb{Z}^{\mathbb{I}}=\oplus_{i \in \mathbb{I}} \mathbb{Z} \alpha_{i}$ via the group isomorphism $\mathbb{Z}^{I} \rightarrow \oplus_{i \in I} \mathbb{Z} t_{i}$ defined by $\alpha_{i} \mapsto t_{i}$, and using this isomorphism we can define $s: \oplus_{i \in I} \mathbb{Z} t_{i} \rightarrow \oplus_{i \in I} \mathbb{Z} t_{i}$ for each $s \in \operatorname{Aut}\left(\mathbb{Z}^{I}\right)$. It can be extended to a unique algebra map $f_{s}: \mathbb{K} \rightarrow \mathbb{K}$ such that $f_{s}\left(t_{i}\right)=s\left(t_{i}\right)$ for all $i \in I$. In this way we obtain an action of $\operatorname{Aut}\left(\mathbb{Z}^{I}\right)$ on $\mathbb{K}$ by algebra maps.

For $x \in \mathcal{X}, i \in I$ and $t \in \mathbb{K}$, let

$$
h_{i}^{x}(t):= \begin{cases}\mathrm{e}^{x}+t \mathrm{n}_{i}^{x} & \text { if } \rho_{i}(x)=x \\ \operatorname{tn}_{i}^{x} & \text { if } \rho_{i}(x) \neq x .\end{cases}
$$

Lemma 4.7. Let $x \in \mathcal{X}$ be such that $\rho_{1}(x)=\rho_{2}(x)=x$ and $d=m_{12}^{x}$ is finite. For $n \in \mathbb{N}_{0}$ set $i_{2 n}=2, i_{2 n+1}=1, j_{2 n}=1, j_{2 n+1}=2$. Then

$$
\begin{aligned}
& h_{i_{1}}^{x}\left(t_{i_{1}}\right) h_{i_{2}}^{x}\left(s_{i_{1}}^{x} \cdot t_{i_{2}}\right) \cdots h_{i_{d}}^{x}\left(s_{i_{1}}^{x} \cdots s_{i_{d-1}} \cdot t_{i_{d}}\right)= \\
& =h_{j_{1}}^{x}\left(t_{j_{1}}\right) h_{j_{2}}^{x}\left(s_{j_{1}}^{x} \cdot t_{j_{2}}\right) \cdots h_{j_{d}}^{x}\left(s_{j_{1}}^{x} \cdots s_{j_{d-1}} \cdot t_{j_{d}}\right) .
\end{aligned}
$$

Proof. By hypothesis $\Delta^{x} \cap\left\{\mathbb{Z} \alpha_{1}+\mathbb{Z} \alpha_{2}\right\}$ is a finite root system, so it corresponds to a finite Cartan matrix $C=\left(\begin{array}{cc}2 & c_{12}^{x} \\ c_{21}^{x} & 2\end{array}\right)$. We have four possibilities.

(i) $C$ is of type $A_{1} \times A_{1}$. Notice that

$$
\begin{aligned}
h_{1}^{x}\left(t_{1}\right) h_{2}^{x}\left(t_{2}\right) & =\mathrm{e}^{x}+t_{1} n_{1}^{x}+t_{2} n_{2}^{x}+t_{1} t_{2} n_{1}^{x} n_{2}^{x} \\
& =\mathrm{e}^{x}+t_{1} n_{1}^{x}+t_{2} n_{2}^{x}+t_{1} t_{2} n_{2}^{x} n_{1}^{x}=h_{2}^{x}\left(t_{2}\right) h_{1}^{x}\left(t_{1}\right) .
\end{aligned}
$$

(ii) $C$ is of type $A_{2}$. Then we compute

$$
\begin{aligned}
& h_{1}^{x}\left(t_{1}\right) h_{2}^{x}\left(t_{1}+t_{2}\right) h_{1}^{x}\left(t_{2}\right)=\left(\mathrm{e}^{x}+t_{1} \mathrm{n}_{1}^{x}\right)\left(\mathrm{e}^{x}+\left(t_{1}+t_{2}\right) \mathrm{n}_{2}^{x}\right)\left(\mathrm{e}^{x}+t_{2} \mathrm{n}_{1}^{x}\right) \\
& =\mathrm{e}^{x}+\left(t_{1}+t_{2}\right) \mathrm{n}_{1}^{x}+\left(t_{1}+t_{2}\right) \mathrm{n}_{2}^{x}+t_{1}\left(t_{1}+t_{2}\right) \mathrm{n}_{1}^{x} \mathrm{n}_{2}^{x} \\
& \quad+\left(t_{1}+t_{2}\right) t_{2} \mathrm{n}_{2}^{x} \mathrm{n}_{1}^{x}+t_{1}\left(t_{1}+t_{2}\right) t_{2} \mathrm{n}_{1}^{x} \mathrm{n}_{2}^{x} \mathrm{n}_{1}^{x} \\
& =\left(\mathrm{e}^{x}+t_{2} \mathrm{n}_{2}^{x}\right)\left(\mathrm{e}^{x}+\left(t_{1}+t_{2}\right) \mathrm{n}_{1}^{x}\right)\left(\mathrm{e}^{x}+t_{1} \mathrm{n}_{2}^{x}\right)=h_{2}^{x}\left(t_{2}\right) h_{1}^{x}\left(t_{1}+t_{2}\right) h_{2}^{x}\left(t_{1}\right) .
\end{aligned}
$$

(iii) $C$ is of type $B_{2}$. Then we compute

$$
\begin{aligned}
h_{1}^{x}\left(t_{1}\right) h_{2}^{x}\left(2 t_{1}+t_{2}\right) h_{1}^{x}\left(t_{1}+t_{2}\right) h_{2}^{x}\left(t_{2}\right)= & \\
= & \left(\mathrm{e}^{x}+t_{1} \mathrm{n}_{1}^{x}\right)\left(\mathrm{e}^{x}+\left(2 t_{1}+t_{2}\right) \mathrm{n}_{2}^{x}\right)\left(\mathrm{e}^{x}+\left(t_{1}+t_{2}\right) \mathrm{n}_{1}^{x}\right)\left(\mathrm{e}^{x}+t_{2} \mathrm{n}_{2}^{x}\right) \\
= & \mathrm{e}^{x}+\left(2 t_{1}+t_{2}\right) \mathrm{n}_{1}^{x}+2\left(t_{1}+t_{2}\right) \mathrm{n}_{2}^{x}+\left(2 t_{1}+t_{2}\right)\left(t_{1}+t_{2}\right) \mathrm{n}_{1}^{x} \mathrm{n}_{2}^{x} \\
& \quad+\left(2 t_{1}+t_{2}\right)\left(t_{1}+t_{2}\right) \mathrm{n}_{2}^{x} \mathrm{n}_{1}^{x}+t_{1}\left(2 t_{1}+t_{2}\right)\left(t_{1}+t_{2}\right) \mathrm{n}_{1}^{x} \mathrm{n}_{2}^{x} \mathrm{n}_{1}^{x} \\
& \quad+\left(2 t_{1}+t_{2}\right)\left(t_{1}+t_{2}\right) t_{2} \mathrm{n}_{2}^{x} \mathrm{n}_{1}^{x} \mathrm{n}_{2}^{x}+t_{1}\left(2 t_{1}+t_{2}\right)\left(t_{1}+t_{2}\right) t_{2} \mathrm{n}_{1}^{x} \mathrm{n}_{2}^{x} \mathrm{n}_{1}^{x} \mathrm{n}_{2}^{x} \\
= & \left(\mathrm{e}^{x}+t_{2} \mathrm{n}_{2}^{x}\right)\left(\mathrm{e}^{x}+\left(t_{1}+t_{2}\right) \mathrm{n}_{1}^{x}\right)\left(\mathrm{e}^{x}+\left(2 t_{1}+t_{2}\right) \mathrm{n}_{2}^{x}\right)\left(\mathrm{e}^{x}+t_{1} \mathrm{n}_{1}^{x}\right) \\
= & h_{2}^{x}\left(t_{2}\right) h_{1}^{x}\left(t_{1}+t_{2}\right) h_{2}^{x}\left(2 t_{1}+t_{2}\right) h_{1}^{x}\left(t_{1}\right) .
\end{aligned}
$$

(iv) $C$ is of type $G_{2}$. Then we compute

$$
\begin{aligned}
h_{1}^{x}\left(t_{1}\right) h_{2}^{x}\left(3 t_{1}\right. & \left.+t_{2}\right) h_{1}^{x}\left(2 t_{1}+t_{2}\right) h_{2}^{x}\left(3 t_{1}+2 t_{2}\right) h_{1}^{x}\left(t_{1}+t_{2}\right) h_{2}^{x}\left(t_{2}\right) \\
=1 & +2\left(2 t_{1}+t_{2}\right) \mathrm{n}_{1}^{x}+2\left(3 t_{1}+2 t_{2}\right) \mathrm{n}_{2}^{x} \\
& +2\left(2 t_{1}+t_{2}\right)\left(3 t_{1}+2 t_{2}\right)\left(\mathrm{n}_{1}^{x} \mathrm{n}_{2}^{x}+\mathrm{n}_{2}^{x} \mathrm{n}_{1}^{x}\right) \\
& +\left(3 t_{1}+t_{2}\right)\left(2 t_{1}+t_{2}\right)\left(3 t_{1}+2 t_{2}\right)\left(\mathrm{n}_{1}^{x} \mathrm{n}_{2}^{x} \mathrm{n}_{1}^{x}+3 \mathrm{n}_{2}^{x} \mathrm{n}_{1}^{x} \mathrm{n}_{2}^{x}\right)
\end{aligned}
$$




$$
\begin{aligned}
& \quad+\left(3 t_{1}+t_{2}\right)\left(2 t_{1}+t_{2}\right)\left(3 t_{1}+2 t_{2}\right)\left(t_{1}+t_{2}\right)\left(\mathrm{n}_{1}^{x} \mathrm{n}_{2}^{x} \mathrm{n}_{1}^{x} \mathrm{n}_{2}^{x}+\mathrm{n}_{2}^{x} \mathrm{n}_{1}^{x} \mathrm{n}_{2}^{x} \mathrm{n}_{1}^{x}\right) \\
& \quad+t_{1}\left(3 t_{1}+t_{2}\right)\left(2 t_{1}+t_{2}\right)\left(3 t_{1}+2 t_{2}\right)\left(t_{1}+t_{2}\right) \mathrm{n}_{1}^{x} \mathrm{n}_{2}^{x} \mathrm{n}_{1}^{x} \mathrm{n}_{2}^{x} \mathrm{n}_{1}^{x} \\
& \quad+\left(3 t_{1}+t_{2}\right)\left(2 t_{1}+t_{2}\right)\left(3 t_{1}+2 t_{2}\right)\left(t_{1}+t_{2}\right) t_{2} \mathrm{n}_{2}^{x} \mathrm{n}_{1}^{x} \mathrm{n}_{2}^{x} \mathrm{n}_{1}^{x} \mathrm{n}_{2}^{x} \\
& \quad+t_{1}\left(3 t_{1}+t_{2}\right)\left(2 t_{1}+t_{2}\right)\left(3 t_{1}+2 t_{2}\right)\left(t_{1}+t_{2}\right) t_{2} \mathrm{n}_{1}^{x} \mathrm{n}_{2}^{x} \mathrm{n}_{1}^{x} \mathrm{n}_{2}^{x} \mathrm{n}_{1}^{x} \mathrm{n}_{2}^{x} \\
& =h_{2}^{x}\left(t_{2}\right) h_{1}^{x}\left(t_{1}+t_{2}\right) h_{2}^{x}\left(3 t_{1}+2 t_{2}\right) h_{1}^{x}\left(2 t_{1}+t_{2}\right) h_{2}^{x}\left(3 t_{1}+t_{2}\right) h_{1}^{x}\left(t_{1}\right),
\end{aligned}
$$

which completes the proof.

Now we consider rank 2 Weyl groupoids with more than one point. Let $m=|\mathcal{X}|$, and assume that the object change diagram has the form:

$$
\bullet x_{1} \stackrel{2}{x_{2}} \frac{1}{x^{x_{3}}} \frac{2}{\ldots} \bullet^{x_{m-1}} \frac{j_{m-1}}{\bullet^{x_{m}},}
$$

where $j_{2 n}=1, j_{2 n+1}=2, x_{n+1}=\rho_{j_{n}}\left(x_{n}\right), n \in \mathbb{Z}$; notice that $x_{k}=x_{2 r m+k}=$ $x_{2 r m-k+1}$ for $0 \leq k \leq m$ and $r \in \mathbb{Z}$. Set also $i_{2 n}=2, i_{2 n+1}=1$.

Given an object $x$ of a Weyl groupoid of rank 2, we set

$$
P_{+}^{x}:=\prod_{n \alpha_{1}+m \alpha_{2} \in \Delta_{+}^{x}}\left(n t_{1}+m t_{2}\right) \in \mathbb{N}_{0}\left[t_{1}, t_{2}\right] .
$$

For each $\alpha \in \Delta_{+}^{x}$ we set also

$$
P_{+}^{x}(\alpha):=\prod_{n \alpha_{1}+m \alpha_{2} \in \Delta_{+}^{x} \backslash\{\alpha\}}\left(n t_{1}+m t_{2}\right) \in \mathbb{N}_{0}\left[t_{1}, t_{2}\right] .
$$

Lemma 4.8. For $x=x_{1}$,

$$
\begin{aligned}
h_{1}^{x_{0}}\left(t_{1}\right) & h_{2}^{x_{1}}\left(s_{1}^{x_{1}} \cdot t_{2}\right) \cdots h_{i_{d}}^{x_{d-1}}\left(s_{i_{1}}^{x_{1}} \cdots s_{i_{d-1}} \cdot t_{i_{d}}\right) \\
& =P_{+}^{x_{1}} \mathrm{n}_{1}^{x_{0}} \mathrm{n}_{2}^{x_{1}} \cdots \mathrm{n}_{i_{d}}^{x_{d-1}}+P_{+}^{x_{1}}\left(\alpha_{1}\right) \mathrm{n}_{2}^{x_{1}} \cdots \mathrm{n}_{i_{d}}^{x_{d-1}} \\
& =h_{2}^{x_{1}}\left(t_{2}\right) h_{1}^{x_{2}}\left(s_{2}^{x_{1}} \cdot t_{1}\right) \cdots h_{j_{d}}^{x_{d}}\left(s_{j_{1}}^{x_{1}} \cdots s_{j_{d-1}} \cdot t_{j_{d}}\right) .
\end{aligned}
$$

For $x=x_{k}, 1<k<m$ even,

$$
\begin{gathered}
h_{1}^{x_{k}}\left(t_{1}\right) h_{2}^{x_{k+1}}\left(s_{1}^{x_{k}} \cdot t_{2}\right) \cdots h_{i_{d}}^{x_{k+d-1}}\left(s_{i_{1}}^{x_{k}} \cdots s_{i_{d-1}} \cdot t_{i_{d}}\right)=P_{+}^{x_{k}} \mathrm{n}_{1}^{x_{k}} \mathrm{n}_{2}^{x_{k+1}} \cdots \mathrm{n}_{i_{d}}^{x_{k+d-1}} \\
=h_{2}^{x_{k}}\left(t_{2}\right) h_{1}^{x_{k-1}}\left(s_{2}^{x_{k}} \cdot t_{1}\right) \cdots h_{j_{d}}^{x_{k-d+1}}\left(s_{j_{1}}^{x_{k}} \cdots s_{j_{d-1}} \cdot t_{j_{d}}\right) .
\end{gathered}
$$

Proof. Let $\beta_{\ell}=s_{1}^{x_{k}} s_{2} \ldots s_{i_{\ell-1}}\left(\alpha_{i_{\ell}}\right)$; in particular $\beta_{1}=\alpha_{1}, \beta_{d}=\alpha_{2}, \Delta_{+}^{a_{k}}=$ $\left\{\beta_{\ell}: 1 \leq \ell \leq d\right\}$ and $\beta_{1}<\beta_{2}<\cdots<\beta_{d}$ is the convex order corresponding to the reduced expression $w=s_{1}^{x_{k}} s_{2} \cdots s_{i_{d}}$. But $\beta_{d}<\beta_{d-1}<\cdots<\beta_{1}$ is also a convex order so it corresponds to another reduced expression of $w$ by $\mathrm{A}$ ]: the unique possible one starting with $\delta_{1}=\alpha_{2}$ is $w=s_{2}^{x_{k}} s_{1} \ldots s_{j_{d}}$. That is, if $\delta_{\ell}=s_{2}^{x_{k}} s_{1} \ldots s_{j_{\ell-1}}\left(\alpha_{j_{\ell}}\right)$, then $\delta_{\ell}=\beta_{d-\ell+1}$.

Notice that $m \mid d$ since $\left(\rho_{1} \rho_{2}\right)^{d}\left(x_{k}\right)=x_{k}$ for all $1 \leq k \leq m$.

If $1<k<m$ and $k$ is even, then

$$
h_{1}^{x_{k}}\left(t_{1}\right)=t_{1} \mathrm{n}_{1}^{x_{k}}, \quad h_{i_{d}}^{x_{k+d-1}}\left(s_{i_{1}}^{x_{k}} \cdots s_{i_{d-1}} \cdot t_{i_{d}}\right)=t_{2} \mathrm{n}_{i_{d}}^{x_{k+d-1}} .
$$

Using the defining relations of $\mathcal{N}$ in (8) we see that

$$
h_{1}^{x_{k}}\left(t_{1}\right) h_{2}^{x_{k+1}}\left(s_{1}^{x_{k}} \cdot t_{1}\right) \cdots h_{i_{d}}^{x_{k+d-1}}\left(s_{i_{1}}^{x_{k}} \cdots s_{i_{d-1}} \cdot t_{i_{d}}\right)=P_{+}^{x_{k}} \mathrm{n}_{1}^{x_{k}} \mathrm{n}_{2}^{x_{k+1}} \cdots \mathrm{n}_{i_{d}}^{x_{k+d-1}},
$$


since $\mathrm{n}_{i}^{x_{s}} \mathrm{n}_{j}^{x_{t}}=\left(\mathrm{n}_{i}^{x_{s}} \mathrm{e}_{i}^{\rho_{i}\left(x_{s}\right)}\right)\left(\mathrm{e}_{j}^{x_{t}} \mathrm{n}_{j}^{x_{t}}\right)=\delta_{\rho_{i}\left(x_{s}\right), x_{t}} \mathrm{n}_{i}^{x_{s}} \mathrm{n}_{j}^{x_{t}}$. Analogously,

$$
h_{2}^{x_{k}}\left(t_{2}\right) h_{1}^{x_{k-1}}\left(s_{2}^{x_{k}} \cdot t_{2}\right) \cdots h_{j_{d}}^{x_{k-d+1}}\left(s_{j_{1}}^{x_{k}} \cdots s_{j_{d-1}} \cdot t_{j_{d}}\right)=P_{+}^{x_{k}} \mathrm{n}_{2}^{x_{k}} \mathrm{n}_{1}^{x_{k-1}} \cdots \mathrm{n}_{j_{d}}^{x_{k-d+1}} \text {. }
$$

By (91), $\mathrm{n}_{1}^{x_{k}} \mathrm{n}_{2}^{x_{k+1}} \cdots \mathrm{n}_{i_{d}}^{x_{k+d-1}}=\mathrm{n}_{2}^{x_{k}} \mathrm{n}_{1}^{x_{k-1}} \cdots \mathrm{n}_{j_{d}}^{x_{k-d+1}}$, so the equality between both expressions follows.

For the first equality, notice that $h_{i_{d}}^{x_{d-1}}\left(s_{i_{1}}^{x_{1}} \cdots s_{i_{d-1}} \cdot t_{i_{d}}\right)=h_{i_{d}}^{x_{d-1}}\left(t_{2}\right)=t_{2} \mathrm{n}_{i_{d}}^{x_{d-1}}$ since $m \mid d$, and $h_{1}^{x_{1}}\left(t_{1}\right)=\mathrm{e}^{x_{1}}+t_{1} \mathrm{n}_{1}^{x_{1}}$, so the equality

$$
h_{1}^{x_{0}}\left(t_{1}\right) \cdots h_{i_{d}}^{x_{d-1}}\left(s_{i_{1}}^{x_{1}} \cdots s_{i_{d-1}} \cdot t_{i_{d}}\right)=P_{+}^{x_{1}} \mathrm{n}_{1}^{x_{0}} \mathrm{n}_{2}^{x_{1}} \cdots \mathrm{n}_{i_{d}}^{x_{d-1}}+P_{+}^{x_{1}}\left(\alpha_{1}\right) \mathrm{n}_{2}^{x_{1}} \cdots \mathrm{n}_{i_{d}}^{x_{d-1}}
$$

follows again by (8). Analogously

$$
h_{2}^{x_{1}}\left(t_{2}\right) \cdots h_{j_{d}}^{x_{d}}\left(s_{j_{1}}^{x_{2}} \cdots s_{j_{d-1}} \cdot t_{j_{d}}\right)=P_{+}^{x_{1}} \mathrm{n}_{2}^{x_{1}} \mathrm{n}_{1}^{x_{2}} \cdots \mathrm{n}_{j_{d}}^{x_{d}}+P_{+}^{x_{1}}\left(\alpha_{1}\right) \mathrm{n}_{2}^{x_{1}} \cdots \mathrm{n}_{j_{d-1}}^{x_{d-1}},
$$

since $h_{j_{d}}^{x_{d}}\left(s_{j_{1}}^{x_{1}} \cdots s_{j_{d-1}} \cdot t_{j_{d}}\right)=\mathrm{e}^{x_{d}}+t_{1} \mathrm{n}_{j_{d}}^{x_{d}}$ and $h_{1}^{x_{2}}\left(t_{2}\right)=t_{2} \mathrm{n}_{2}^{x_{1}}$. Finally the equality between both expressions follows again by (9).

Example 4.9. For $x_{1}$ as in (6)

$$
\begin{aligned}
h_{1}^{x_{1}}\left(t_{1}\right) h_{2}^{x_{1}}\left(t_{1}+t_{2}\right) h_{1}^{x_{2}}\left(t_{2}\right) & =\left(t_{1}+t_{2}\right) t_{2} \mathrm{n}_{2}^{x_{1}} \mathrm{n}_{1}^{x_{2}}+t_{1}\left(t_{1}+t_{2}\right) t_{2} \mathrm{n}_{1}^{x_{1}} \mathrm{n}_{2}^{x_{1}} \mathrm{n}_{1}^{x_{2}} \\
& =h_{2}^{x_{1}}\left(t_{2}\right) h_{1}^{x_{2}}\left(t_{1}+t_{2}\right) h_{2}^{x_{3}}\left(t_{1}\right)
\end{aligned}
$$

and for $x_{2}$,

$$
\begin{aligned}
h_{1}^{x_{2}}\left(t_{1}\right) h_{2}^{x_{3}}\left(t_{1}+t_{2}\right) h_{1}^{x_{3}}\left(t_{2}\right) & =t_{1}\left(t_{1}+t_{2}\right) t_{2} \mathrm{n}_{1}^{x_{2}} \mathrm{n}_{2}^{x_{3}} \mathrm{n}_{1}^{x_{3}} \\
& =h_{2}^{x_{2}}\left(t_{2}\right) h_{1}^{x_{1}}\left(t_{1}+t_{2}\right) h_{2}^{x_{1}}\left(t_{1}\right) .
\end{aligned}
$$

Now for $x_{1}$ as in (7) we have that

$$
\begin{aligned}
h_{1}^{x_{1}}\left(t_{1}\right) & h_{2}^{x_{1}}\left(2 t_{1}+t_{2}\right) h_{1}^{x_{2}}\left(t_{1}+t_{2}\right) h_{2}^{x_{2}}\left(t_{2}\right)= \\
& =\left(2 t_{1}+t_{2}\right)\left(t_{1}+t_{2}\right) t_{2} \mathrm{n}_{2}^{x_{1}} \mathrm{n}_{1}^{x_{2}} \mathrm{n}_{2}^{x_{2}}+t_{1}\left(2 t_{1}+t_{2}\right)\left(t_{1}+t_{2}\right) t_{2} \mathrm{n}_{1}^{x_{1}} \mathrm{n}_{2}^{x_{1}} \mathrm{n}_{1}^{x_{2}} \mathrm{n}_{2}^{x_{2}} \\
& =h_{2}^{x_{1}}\left(t_{2}\right) h_{1}^{x_{2}}\left(t_{1}+t_{2}\right) h_{2}^{x_{2}}\left(2 t_{1}+t_{2}\right) h_{1}^{x_{1}}\left(t_{1}\right) .
\end{aligned}
$$

Notice the difference with these cases of standard Weyl groupoids with more than one point and those with a unique $x$ in Lemma 4.7, although they have the same sets of roots and the same finite Cartan matrices (of type $A_{2}, B_{2}$ respectively), the expressions of products in Lemmas 4.7 and 4.8 differ.

Example 4.10. We compute the expression in Lemma4.8 for the Weyl groupoid of the Nichols algebra [H2, Table 1, Row 10]. Its Cartan graph is

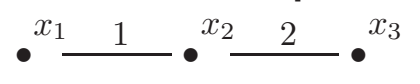

for the following Cartan matrices:

$$
C_{1}=\left[\begin{array}{cc}
2 & -2 \\
-2 & 2
\end{array}\right], \quad C_{2}=\left[\begin{array}{cc}
2 & -2 \\
-1 & 2
\end{array}\right], \quad C_{3}=\left[\begin{array}{cc}
2 & -4 \\
-1 & 2
\end{array}\right] .
$$

For $x_{1}$ we have that

$$
\begin{aligned}
h_{1}^{x_{1}}\left(t_{1}\right) & h_{2}^{x_{2}}\left(2 t_{1}+t_{2}\right) h_{1}^{x_{3}}\left(t_{1}+t_{2}\right) h_{2}^{x_{3}}\left(2 t_{1}+3 t_{2}\right) h_{1}^{x_{2}}\left(t_{1}+2 t_{2}\right) h_{2}^{x_{1}}\left(t_{2}\right) \\
& =P_{+}^{x_{1}} \mathrm{n}_{1}^{x_{1}} \mathrm{n}_{2}^{x_{2}} \mathrm{n}_{1}^{x_{3}} \mathrm{n}_{2}^{x_{3}} \mathrm{n}_{1}^{x_{2}} \mathrm{n}_{2}^{x_{1}}+P_{+}^{x_{1}}\left(\alpha_{2}\right) \mathrm{n}_{1}^{x_{1}} \mathrm{n}_{2}^{x_{2}} \mathrm{n}_{1}^{x_{3}} \mathrm{n}_{2}^{x_{3}} \mathrm{n}_{1}^{x_{2}} \\
& =P_{+}^{x_{1}} \mathrm{n}_{2}^{x_{1}} \mathrm{n}_{1}^{x_{1}} \mathrm{n}_{2}^{x_{2}} \mathrm{n}_{1}^{x_{3}} \mathrm{n}_{2}^{x_{3}} \mathrm{n}_{1}^{x_{2}}+P_{+}^{x_{1}}\left(\alpha_{2}\right) \mathrm{n}_{1}^{x_{1}} \mathrm{n}_{2}^{x_{2}} \mathrm{n}_{1}^{x_{3}} \mathrm{n}_{2}^{x_{3}} \mathrm{n}_{1}^{x_{2}} \\
& =h_{2}^{x_{1}}\left(t_{2}\right) h_{1}^{x_{1}}\left(t_{1}+2 t_{2}\right) h_{2}^{x_{2}}\left(2 t_{1}+3 t_{2}\right) h_{1}^{x_{3}}\left(t_{1}+t_{2}\right) h_{2}^{x_{3}}\left(2 t_{1}+t_{2}\right) h_{1}^{x_{2}}\left(t_{1}\right) .
\end{aligned}
$$


For $x_{2}$ we compute

$$
\begin{aligned}
& h_{1}^{x_{2}}\left(t_{1}\right) h_{2}^{x_{1}}\left(2 t_{1}+t_{2}\right) h_{1}^{x_{1}}\left(3 t_{1}+2 t_{2}\right) h_{2}^{x_{2}}\left(4 t_{1}+3 t_{2}\right) h_{1}^{x_{3}}\left(t_{1}+t_{2}\right) h_{2}^{x_{3}}\left(t_{2}\right) \\
& \quad=P_{+}^{x_{2}} \mathrm{n}_{1}^{x_{2}} \mathrm{n}_{2}^{x_{1}} \mathrm{n}_{1}^{x_{1}} \mathrm{n}_{2}^{x_{2}} \mathrm{n}_{1}^{x_{3}} \mathrm{n}_{2}^{x_{3}}=P_{+}^{x_{2}} \mathrm{n}_{2}^{x_{2}} \mathrm{n}_{1}^{x_{3}} \mathrm{n}_{2}^{x_{3}} \mathrm{n}_{1}^{x_{2}} \mathrm{n}_{2}^{x_{1}} \mathrm{n}_{1}^{x_{1}} \\
& \quad=h_{2}^{x_{2}}\left(t_{2}\right) h_{1}^{x_{3}}\left(t_{1}+t_{2}\right) h_{2}^{x_{3}}\left(4 t_{1}+3 t_{2}\right) h_{1}^{x_{2}}\left(3 t_{1}+2 t_{2}\right) h_{2}^{x_{1}}\left(2 t_{1}+t_{2}\right) h_{1}^{x_{1}}\left(t_{1}\right) .
\end{aligned}
$$

Finally for $x_{3}$ we have that

$$
\begin{aligned}
h_{1}^{x_{3}}\left(t_{1}\right) & h_{2}^{x_{3}}\left(4 t_{1}+t_{2}\right) h_{1}^{x_{2}}\left(3 t_{1}+t_{2}\right) h_{2}^{x_{1}}\left(2 t_{1}+t_{2}\right) h_{1}^{x_{1}}\left(t_{1}+t_{2}\right) h_{2}^{x_{2}}\left(t_{2}\right) \\
& =P_{+}^{x_{3}} \mathrm{n}_{1}^{x_{3}} \mathrm{n}_{2}^{x_{3}} \mathrm{n}_{1}^{x_{2}} \mathrm{n}_{2}^{x_{1}} \mathrm{n}_{1}^{x_{1}} \mathrm{n}_{2}^{x_{2}}+P_{+}^{x_{3}}\left(\alpha_{1}\right) \mathrm{n}_{2}^{x_{3}} \mathrm{n}_{1}^{x_{2}} \mathrm{n}_{2}^{x_{1}} \mathrm{n}_{1}^{x_{1}} \mathrm{n}_{2}^{x_{2}} \\
& =P_{+}^{x_{3}} \mathrm{n}_{2}^{x_{3}} \mathrm{n}_{1}^{x_{2}} \mathrm{n}_{2}^{x_{1}} \mathrm{n}_{1}^{x_{1}} \mathrm{n}_{2}^{x_{2}} \mathrm{n}_{1}^{x_{3}}+P_{+}^{x_{3}}\left(\alpha_{1}\right) \mathrm{n}_{2}^{x_{3}} \mathrm{n}_{1}^{x_{2}} \mathrm{n}_{2}^{x_{1}} \mathrm{n}_{1}^{x_{1}} \mathrm{n}_{2}^{x_{2}} \\
& =h_{2}^{x_{3}}\left(t_{2}\right) h_{1}^{x_{2}}\left(t_{1}+t_{2}\right) h_{2}^{x_{1}}\left(2 t_{1}+t_{2}\right) h_{1}^{x_{1}}\left(3 t_{1}+t_{2}\right) h_{2}^{x_{2}}\left(4 t_{1}+t_{2}\right) h_{1}^{x_{3}}\left(t_{1}\right) .
\end{aligned}
$$

Proposition 4.11. Fix $i \neq j, x \in \mathcal{X}$. For $n \in \mathbb{N}_{0}$, set $m=m_{i j}^{x}$,

$$
\begin{array}{lll}
i_{2 n}=j, & i_{2 n+1}=i, & x_{n}=\rho_{i_{n-1}} \cdots \rho_{i_{1}}(x), \\
j_{2 n}=i, & j_{2 n+1}=j, & y_{n}=\rho_{i_{n-1}} \cdots \rho_{j_{1}}(x) .
\end{array}
$$

Then

$$
\begin{aligned}
& h_{i_{1}}^{x_{1}}\left(t_{i_{1}}\right) h_{i_{2}}^{x_{2}}\left(s_{i_{1}}^{x_{1}} \cdot t_{i_{2}}\right) \cdots h_{i_{m}}^{x_{m}}\left(s_{i_{1}}^{x_{1}} \cdots s_{i_{m-1}} \cdot t_{i_{m}}\right)= \\
& =h_{j_{1}}^{y_{1}}\left(t_{j_{1}}\right) h_{j_{2}}^{y_{2}}\left(s_{j_{1}}^{y_{1}} \cdot t_{j_{2}}\right) \cdots h_{j_{m}}^{y_{m}}\left(s_{j_{1}}^{y_{1}} \cdots s_{j_{m-1}} \cdot t_{j_{m}}\right) .
\end{aligned}
$$

Proof. If $\rho_{i}(x)=\rho_{j}(x)=x$, then it follows by Lemma 4.7. If either $\rho_{i}(x) \neq x$ or $\rho_{j}(x) \neq x$, and the object change is not a cycle, then it has the shape (12) and the equality follows by Lemma 4.8. Otherwise the object change is a cycle with $d$ objects, where $d \mid m$. Then $h_{i_{\ell}}^{x_{\ell}}(t)=\operatorname{tn}_{i_{\ell}}^{x_{\ell}}$ for all $\ell$ so

$$
\begin{gathered}
h_{i_{1}}^{x_{1}}\left(t_{i_{1}}\right) h_{i_{2}}^{x_{2}}\left(s_{i_{1}}^{x_{1}} \cdot t_{i_{2}}\right) \cdots h_{i_{m}}^{x_{m}}\left(s_{i_{1}}^{x_{1}} \cdots s_{i_{m-1}} \cdot t_{i_{m}}\right)=P_{+}^{x_{1}} \mathrm{n}_{1}^{x_{1}} \mathrm{n}_{2}^{x_{2}} \cdots \mathrm{n}_{i_{m}}^{x_{m}} \\
=h_{j_{1}}^{y_{1}}\left(t_{j_{1}}\right) h_{j_{2}}^{y_{2}}\left(s_{j_{1}}^{y_{1}} \cdot t_{j_{2}}\right) \cdots h_{j_{m}}^{y_{m}}\left(s_{j_{1}}^{y_{1}} \cdots s_{j_{m-1}} \cdot t_{j_{m}}\right),
\end{gathered}
$$

where last equality follows by (9).

Hence we obtain a representation of the groupoid $\widetilde{\mathcal{W}}$, a key step towards our main result, Theorem 5.2 .

Theorem 4.12. There exists a well-defined map $\Psi: \widetilde{\mathcal{W}} \rightarrow \operatorname{End}(\mathbb{K} \mathcal{N})$ such that

$$
\Psi\left(\widetilde{\sigma}_{i_{1}}^{x} \cdots \widetilde{\sigma}_{i_{m}}\right)\left(f T_{w}\right):=h_{i_{1}}^{x_{1}}\left(\beta_{1}\right) h_{i_{2}}^{x_{2}}\left(\beta_{2}\right) \cdots h_{i_{1}}^{x_{m}}\left(\beta_{m}\right)\left(s_{i_{1}}^{x_{1}} \cdots s_{i_{m}}^{x_{m}} \cdot f\right) T_{w},
$$

for each $f \in \mathbb{K}, w \in \mathcal{W}$, where $x_{j}=\rho_{i_{j-1}} \cdots \rho_{i_{1}}(x), \beta_{j}=s_{i_{1}}^{x} \cdots s_{i_{j-1}}\left(\alpha_{i_{j}}\right)$.

Proof. We claim that there exists $\Psi: \widetilde{\mathcal{W}} \rightarrow \operatorname{End}(\mathbb{K} \mathcal{N})$ such that

$$
\Psi\left(\operatorname{id}_{x} \widetilde{\sigma}_{i}\right)\left(f T_{w}\right):=h_{i}^{a}\left(\alpha_{i}\right)\left(s_{i}^{a} \cdot f\right) T_{w}, \quad f \in \mathbb{K}, w \in \mathcal{W},
$$

from which the statement follows. We have to prove that it is well defined. Fix $i \neq j, x \in \mathcal{X}$. Set $m=m_{i j}^{x}$, and the same elements defined in (13). It is enough to prove that

$$
\Psi\left(\widetilde{\sigma}_{i_{1}}^{x} \cdots \widetilde{\sigma}_{i_{d}}\right)=\Psi\left(\tilde{\sigma}_{j_{1}}^{x} \cdots \widetilde{\sigma}_{j_{d}}\right) .
$$

But this equality follows by Proposition 4.11 and [HY1, Lemma 5], since

$$
\begin{aligned}
\Psi\left(\widetilde{\sigma}_{i_{1}}^{x} \cdots \widetilde{\sigma}_{i_{d}}\right)\left(f T_{w}\right):= & h_{i_{1}}^{x_{1}}\left(t_{i_{1}}\right) h_{i_{2}}^{x_{2}}\left(s_{i_{1}}^{x_{1}} \cdot t_{i_{2}}\right) \cdots h_{i_{d}}^{x_{d}}\left(s_{i_{1}}^{x_{1}} \cdots s_{i_{d-1}} \cdot t_{i_{d}}\right) \\
& \left(s_{i_{1}}^{x_{1}} \cdots s_{i_{d}} \cdot f\right) T_{w},
\end{aligned}
$$


and we have the corresponding formula for $\Psi\left(\widetilde{\sigma}_{j_{1}}^{x} \cdots \widetilde{\sigma}_{j_{d}}\right)\left(f T_{w}\right)$.

\section{BRUhat ORDER FOR WEYL GROUPOIDS}

Now we are ready to define the Bruhat order for Weyl groupoids.

Definition 5.1. Let $(\mathcal{X}, \rho, \mathcal{C})$ be a semi-Cartan graph and $\mathcal{R}$ a $\operatorname{GRS}$ of $(\mathcal{X}, \rho, \mathcal{C})$.

Let $x, y \in \mathcal{X}$. For $w \in \mathcal{W}(y, x)$ with $s_{i_{1}}^{x_{1}} s_{i_{2}}^{x_{2}} \cdots s_{i_{r}}^{x_{r}}$ being a reduced expression of $w$. We say that a subsequence $a(1), \ldots, a(t)$ of $i_{1} \ldots, i_{r}$ is $(y, x)$-good if $x_{z+1}=x_{z}$ for $z \in\{1, \ldots, r\} \backslash\{a(1), \ldots, a(t)\}$. Given $u, w \in \mathcal{W}(y, x)$, we say that $u<w$ if there exists a reduced expression $w=s_{i_{1}}^{x_{1}} s_{i_{2}}^{x_{2}} \cdots s_{i_{r}}^{x_{r}}$ and a subsequence $a(1), \ldots, a(t)$ of $i_{1} \ldots, i_{r}$ such that $u=s_{a(1)}^{x} s_{a(2)} \cdots s_{a(t)}$ is a reduced expression of $u$. It defines a partial order on $\mathcal{W}(y, x)$ called the Bruhat order.

Now we are ready to prove that Bruhat order is well-defined.

Theorem 5.2. Let $w \in \mathcal{W}(y, x), x, y \in \mathcal{X}$. Assume that $r:=\ell(w)>0$ and

$$
w=\sigma_{i_{1}}^{x} \cdots \sigma_{i_{r}}=\sigma_{j_{1}}^{x} \cdots s_{j_{r}} .
$$

are two reduced expressions of $w$.

Then for any $(y, x)$-good sub-sequence $a(1), \ldots, a(t)$ of $i_{1}, \ldots, i_{r}$ such that

$$
u:=\sigma_{a(1)}^{x} \cdots \sigma_{a(t)}, \quad \ell(u)=t, u \in \mathcal{W}(y, x),
$$

there exists a $(y, x)$-good sub-sequence $b(1), \ldots, b(t)$ of $j_{1}, \ldots, j_{r}$ such that

$$
u=\sigma_{b(1)}^{x} \cdots \sigma_{b(t)} .
$$

Proof. Fix $w \in \mathcal{W}(y, x)$ such that $r:=\ell(w)>0$, and a reduced expression $w=\sigma_{i_{1}}^{x} \cdots \sigma_{i_{r}}$. Let $\Theta\left(x ; i_{1}, \ldots, i_{r}\right)$ be the set of elements

$$
u:=\sigma_{a(1)}^{x} \cdots \sigma_{a(t)}, \quad \text { satisfies } \ell(u)=t, u \in \mathcal{W}(y, x),
$$

for some $(y, x)$-good sub-sequence $a(1), \ldots, a(t)$ of $i_{1}, \ldots, i_{r}$.

Let $u \in \mathcal{W}, x \in \mathcal{X}, i \in I, m=\ell(u)$. From Theorem 4.4 we see that $n_{i}^{x} T_{u}=0$ if $\ell\left(\sigma_{i}^{x} u\right)<m+1$, and $n_{i}^{x} T_{u}=T_{\sigma_{i}^{x} u}$ if $\ell\left(\sigma_{i}^{x} u\right)=m+1$. Note that

$$
\Psi\left(\tilde{\sigma}_{i_{1}}^{x} \cdots \widetilde{\sigma}_{i_{m}}\right)(1)=h_{i_{1}}^{x_{1}}\left(\beta_{1}\right) h_{i_{2}}^{x_{2}}\left(\beta_{2}\right) \cdots h_{i_{1}}^{x_{m}}\left(\beta_{m}\right),
$$

and all the $\beta_{j}$ are positive roots. Then there exist non-zero polynomials $f_{u} \in$ $\mathbb{Z}\left[t_{i} \mid i \in I\right], u \in \Theta\left(X_{1} ; i_{1}, \ldots, i_{r}\right)$, such that

$$
\Psi\left(\widetilde{\sigma}_{i_{1}}^{x} \cdots \tilde{\sigma}_{i_{m}}\right)(1)=\sum_{u \in \Theta\left(x ; i_{1}, \ldots, i_{r}\right)} f_{u} T_{u} .
$$

Moreover all the polynomials $f_{u}$ have non-negative coefficients.

Therefore the set $\Theta\left(X_{1} ; i_{1}, \ldots, i_{r}\right)$ is identified with the subset of $\mathcal{W}$ such that $T_{u}$ appears with non-zero coefficient in $\Psi\left(\widetilde{\sigma}_{i_{1}}^{x} \cdots \widetilde{\sigma}_{i_{m}}\right)(1)$. But $\Psi\left(\widetilde{\sigma}_{i_{1}}^{x} \cdots \widetilde{\sigma}_{i_{m}}\right)(1)$ does not depend on the reduced expression of $w$, so the statement follows. 


\section{REFERENCES}

[A] I. Angiono, A presentation by generators and relations of Nichols algebras of diagonal type and convex orders on root systems. J. Europ. Math. Soc. 17 (2015), 2643-2671.

[AA] N. Andruskiewitsch, I. Angiono, Weyl groupoids, contragredient Lie superalgebras and Nichols algebras, in preparation.

[AAY] N. Andruskiewitsch, I. Angiono, H. Yamane, On pointed Hopf superalgebras. Contemp. Math. 544 (2011), 123-140.

[AHS] N. Andruskiewitsch, I. Heckenberger, H.-J. Schneider, The Nichols algebra of a semisimple Yetter-Drinfeld module, Amer. J. Math. 132 (2010), 1493-1547.

[AY] I. Angiono and H. Yamane, The R-matrix of quantum doubles of Nichols algebras of diagonal type, J. Math. Phys. 56, 021702 (2015)

[AYY] S. Azam, H. Yamane, M. Yousofzadeh, Classification of Finite Dimensional Irreducible Representations of Generalized Quantum Groups via Weyl Groupoids. Publ. Res. Inst. Math. Sci. 51 (2015), 59-130.

[BS] N. Bergeron, F. Sottile, Schubert polynomials, the Bruhat order, and the geometry of flag manifolds. Duke Math. J. 95 (1998), 373-423.

[CH] M. Cuntz, I. Heckenberger, Finite Weyl groupoids of rank three. Trans. Amer. Math. Soc. 364 (2012), 1369-1393.

[H1] I. Heckenberger, The Weyl groupoid of a Nichols algebra of diagonal type, Invent. Math. 164, 175-188 (2006).

[H2] I. Heckenberger, Classification of arithmetic root systems, Adv. Math. 220 (2009) 59-124.

[HS] I. Heckenberger, H.-J. Schneider, Right coideal subalgebras of Nichols algebras and the Duflo order on the Weyl groupoid. Israel J. Math. 197 (2013), 139-187.

[HY1] I. Heckenberger, H. Yamane, A generalization of Coxeter groups, root systems, and Matsumoto's theorem. Math. Z. 259 (2008), 255-276.

[HY2] I. Heckenberger, H. Yamane, Drinfel'd doubles and Shapovalov determinants. Rev. Un. Mat. Argentina 51 (2010), 107-146.

[HW] I. Heckenberger, V. Welker, Geometric combinatorics of Weyl groupoids. J. Algebraic Combin. 34 (2011), 115-139.

[IN] T. Ikeda, H. Naruse, Excited Young diagrams and equivariant Schubert calculus. Trans. Amer. Math. Soc. 361 (2009), 5193-5221.

[Ka] V. Kac, Infinite-dimensional Lie algebras, 3rd Edition. Cambridge University Press, Cambridge, 1990.

[M] I. Macdonald, Affine Hecke algebras and orthogonal polynomials. Cambridge Tracts in Mathematics, 157. Cambridge University Press, Cambridge, 2003. x+175 pp.

[Se] V. Serganova, On generalizations of root systems. Comm. Algebra 24 (1996), 42814299.

[SV] A. Sergeev, A. Veselov, Grothendieck rings of basic classical Lie superalgebras, Ann. Math. 173 (2011), 663-703.

[Y] H. Yamane, Iwahori-Hecke type algebras associated with the Lie superalgebras $A(m, n), B(m, n), C(n)$ and $D(m, n)$. New trends in combinatorial representation theory, 197-222, RIMS Kokyuroku Bessatsu, B11, Res. Inst. Math. Sci. (RIMS), Kyoto, 2009.

Famaf-Ciem (COniCet), Universidad Nacional de Córdoba, Medina Allende S/n, Ciudad Universitaria (5000) Córdoba, República Argentina.

E-mail address: angiono@famaf.unc.edu.ar

University of Toyama, Faculty of Science, Gofuku 3190, Toyama-shi, Toyama 930-8555, JAPAN

E-mail address: hiroyuki@sci.u-toyama.ac.jp 